\title{
Astronomers grapple with new era of fast radio bursts
}

\section{Signals have progressed from astronomical peculiarity to mainstream research area.}

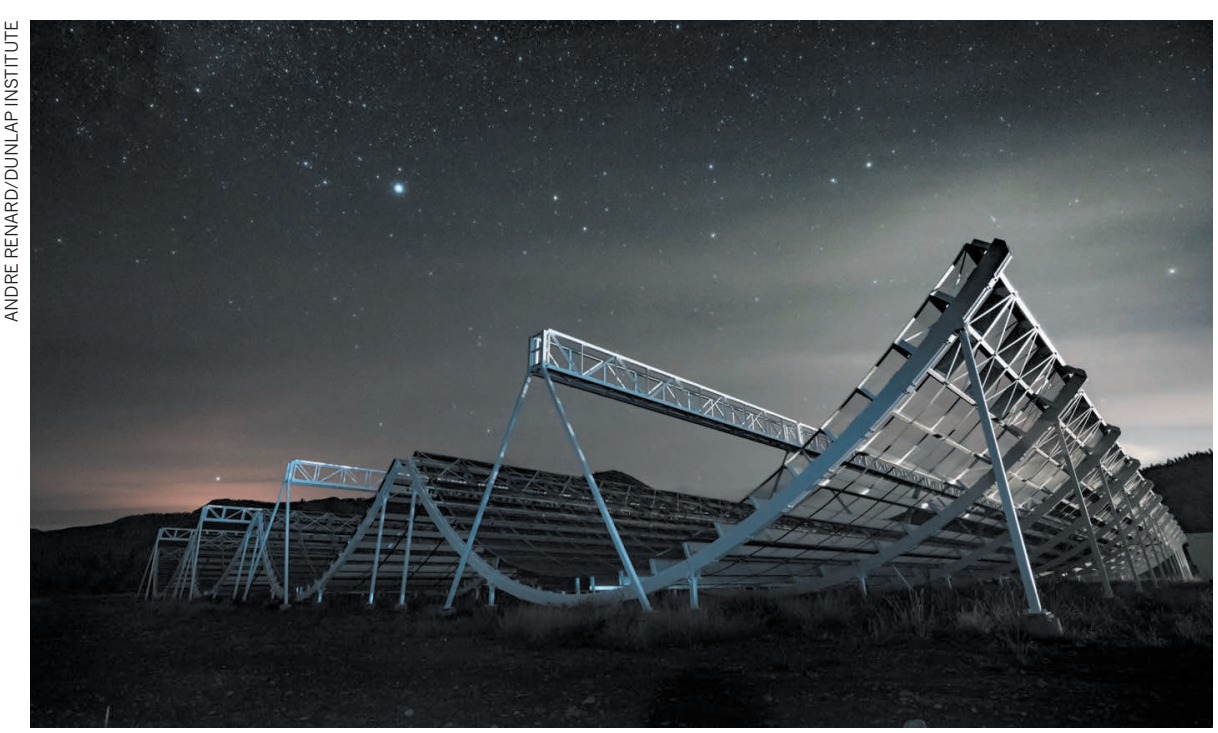

The CHIME radio observatory in Canada will start looking for fast radio bursts this year.

\section{BY ELIZABETH GIBNEY}

$\mathrm{O}$ ne of the most perplexing phenomena in astronomy has come of age. The fleeting blasts of energetic cosmic radiation of unknown cause, now known as fast radio bursts (FRBs), were first detected a decade ago. At the time, many astronomers dismissed the seemingly random blasts as little more than glitches. And although key facts, such as what causes them, are still largely a mystery, FRBs are now accepted as a genuine class of celestial signal and have spawned a field of their own.

The passage was marked this month by the first major meeting on FRBs, held in Aspen, Colorado, on 12-17 February. As well as celebrating a fleet of searches for the signals, the meeting's 80 delegates grappled with how best to design those hunts and pin down the signals' origins and precise distances. The trajectory mirrors that of astronomers 20 years ago when they were getting to grips with $\gamma$-ray bursts, which are now a staple of astronomical observation, says Bing Zhang, a theoretical astrophysicist at the University of Nevada, Las Vegas.

"The meeting has really focused the field a lot," says Sarah Burke Spolaor, an astronomer at West Virginia University in Morgantown. Debates continue over how to root out detection bias and coordinate observations and on what can be learnt by studying patterns in the existing FRB population.

The first FRB was co-discovered ${ }^{1}$ in 2007 by astronomer Duncan Lorimer at West Virginia University. He found in archived pulsar data a 5-millisecond radio frequency burst that was so bright it couldn't be ignored. Astronomers have since seen 25 FRBs. All are brief radio signals, lasting no more than a few thousandths of a second. They seem to come from sources across the sky and beyond our Galaxy. Some last longer than others, and the light from a few is polarized.

A discovery last year caused further excitement. Astronomers reported ${ }^{2}$ that they had found a repeating FRB - a surprise, because all the other signals had been one-off blips. And in January this year, its origin was identified $^{3}$ : a faint, distant dwarf galaxy around 780 megaparsecs ( 2.5 billion light years) away, in a star-forming region that also hums with a steady radio source.

The repeater has gone some way to focusing the FRB field, says Edo Berger, an astronomer at Harvard University in Cambridge, Massachusetts. Astronomers have now observed nearly 200 signals from it; details of 20 have been published. It bolsters the hypothesis that the signals are extragalactic, something most FRB researchers now agree on, and its location is reshaping theories about possible causes.

Dwarf galaxies host fewer stars than most, so tracking an FRB to one is surprising, says Berger. He thinks that the unusual environment is more than coincidence, and that FRBs may come from super-powerful magnetars - dense, magnetic stars thought to form after an abnormally massive explosion, such as an extremely energetic supernova. Studies suggest that such events seem to be more common in dim dwarf galaxies, he says. Others think the bursts might come from active galactic nuclei, regions at the centres of some galaxies that are thought to host supermassive black holes. Streams of plasma from these could comb nearby pulsars to produce FRBs, says Zhang, which could also explain a recent, although tentative, observation of a faint $\gamma$-ray burst coinciding with an FRB.

At the meeting, some astronomers proposed
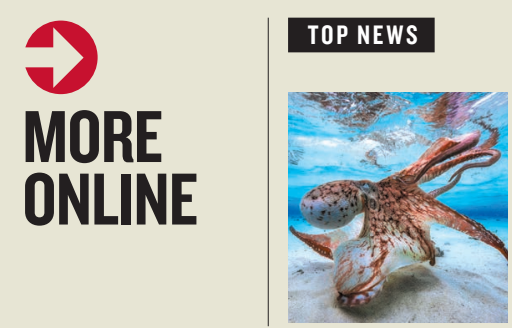

Images of the month: fake stars, panda suits and a dancing octopus go.nature. com/2mab8ue

\section{MORE NEWS}

- These seven alien worlds could help to explain how planets form go.nature.com/2lpkldq

- Bees learn football from their buddies go.nature.com/2lupfu5 - UK universities unlikely to be lured to France go.nature.com/2lpxpwr

\section{NATURE PODCAST}

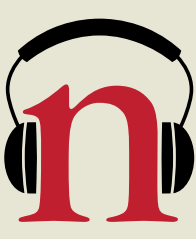

Migration special: a researcher seeks refuge; smart borders; and climate migration nature.com/nature/ podcast 
reversing the search strategy, and looking for FRBs in similarly strange galaxies, as well as trying to locate the origin of single bursts when they occur. And heated debate arose over whether all FRBs are likely to come from the same kind of source as the repeater, and so whether astronomers might detect repeated signals from all FRBs if they look for long enough. "The answer was definitely maybe," says Burke Spolaor. But there could be different kinds of sources, leaving open the question of how much one repeater can teach about FRBs in general, she adds.

\section{CUTTING BIAS}

A major issue is how to avoid bias. The fact that they were discovered by researchers looking for pulsars - small, dense, rotating stars - could bias the generation of theories about FRBs: astronomers might be drawn to models involving objects similar to pulsars. Detection bias is also an issue, in part because many FRB searches are piggy-backed onto those that are optimized for finding sources within the Milky Way that repeat regularly, rather than sporadic extragalactic events. The more astronomers look, the more they find FRBs in unexpected locations and with unusual features.

To ensure that astronomers are seeing a representative sample, they need to look for signals across a broader range of frequencies, says Burke Spolaor. They should also pay more attention to the polarization of FRB light, she adds, which can provide clues about the environment of the source.

About 30 telescopes are looking for FRBs, and dedicated searches are increasing. The conference buzzed with excitement about the Canadian Hydrogen Intensity Mapping Experiment (CHIME), a radio telescope in Canada that should start hunting for FRBs later this year and could see as many as a dozen a day.

But observations need to be better coordinated, says Berger. Delegates planned efforts to automatically release FRB results in real time for follow-up by other telescopes, as is already done for other kinds of fleeting astronomical signal.

Although FRBs remain a mystery, the field has surged forward since Lorimer identified the first burst. The fact that the community now agrees, for instance, that the bursts are extragalactic is a big step forward. Lorimer's wife, West Virginia University astrophysicist Maura McLaughlin, initially doubted they were even extraterrestrial, Lorimer told the meeting. "The community was quite sharply divided about it, even in our own household. We've come a long way since then." -

1. Lorimer, D. R., Bailes, M., McLaughlin, M. A., Narkevic, D. J. \& Crawford, F. Science $\mathbf{3 1 8}$ 777-780 (2007)

2. Spitler, L. G. et al. Nature 531, 202-205 (2016).

3. Chatterjee, S. et al. Nature 541, 58-61 (2017).

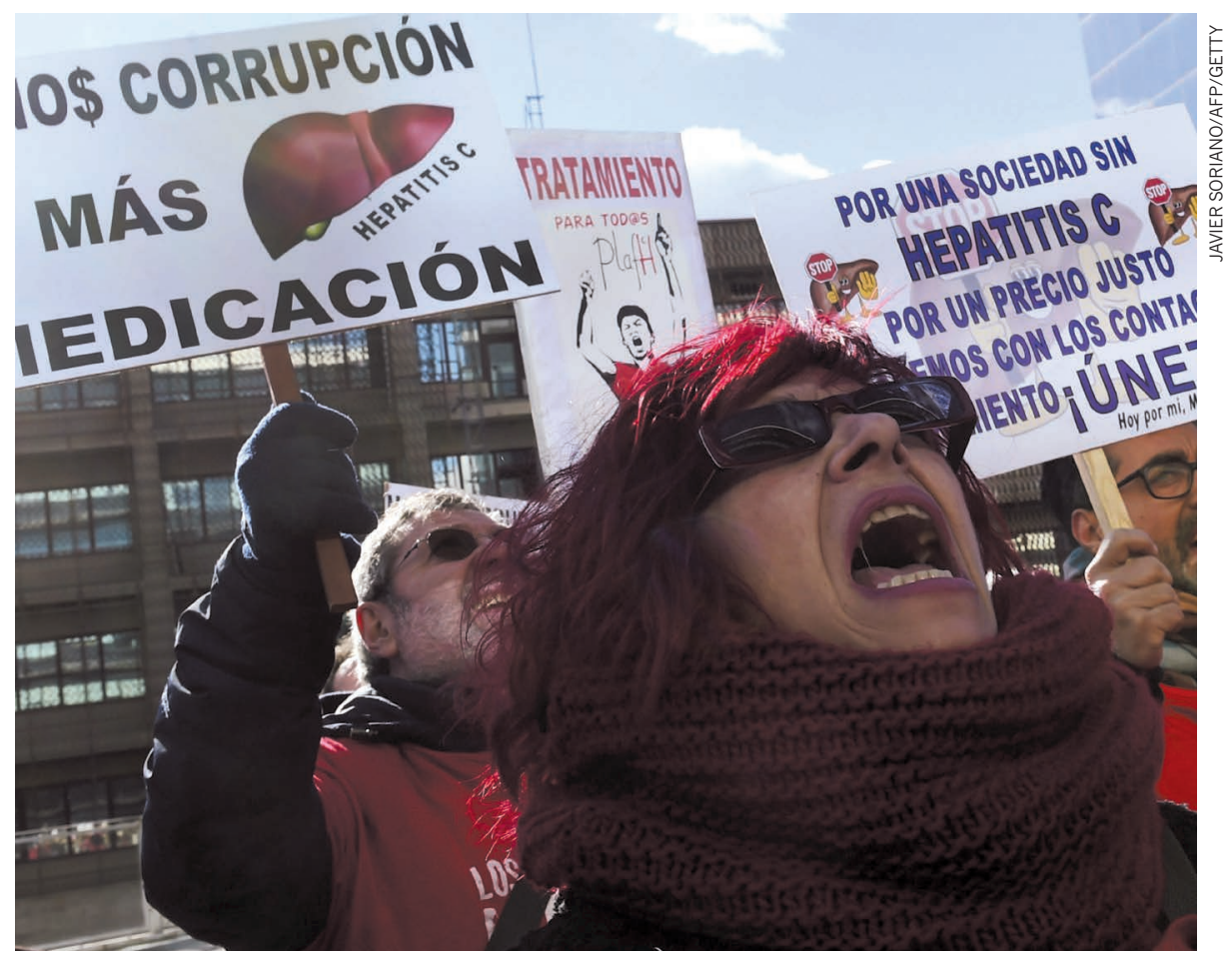

People in Madrid demonstrate against the high price of hepatitis $\mathrm{C}$ drugs.

\section{PUBLIC HEALTH}

\section{Hepatitis C drugs stoke patent fight}

\section{Lawsuits in India and Argentina seek to reduce drug costs.}

\section{BY AMY MAXMEN}

$\mathrm{T}$ The liver disease hepatitis $C$ is the new battleground for lawsuits intended to slash the cost of life-saving medicines. In February alone, five suits were filed in India and Argentina claiming that the latest class of antiviral drugs does not warrant the 20-year patent monopoly that manufacturers have sought in those countries.

In the 2000s, successful challenges to patents on HIV drugs gave poor nations access to highquality 'generic' copies of the medications at rock-bottom prices. Now, buoyed by that success, activists are applying the same strategy to a fresh wave of hepatitis $\mathrm{C}$ drugs. They note that the standard 12-week course of treatment costs more than the average annual salary for millions of people in middle-income countries.

Public-health experts say that expanding access to the drugs would have immediate benefits. Roughly 177.5 million adults worldwide are infected with the hepatitis $C$ virus, which can cause liver cancer and cirrhosis if left untreated - but the latest antiviral medications have revolutionized care. The first to reach the market was sofosbuvir, sold under the name Sovaldi by Gilead Sciences of Foster City, California; clinical trials of the drug in combination with other medications have shown a cure rate of $95 \%$ or more. "If these medicines were made widely available, you could make a plan to eliminate this disease," says Brook Baker, a law specialist at Northeastern University in Boston, Massachusetts.

As the world's main supplier of generic drugs, India is at the centre of the current patent fight. Patient advocates celebrated when the country's patent office rejected Gilead's application for a basic patent on sofosbuvir in January 2015, on the grounds that it was not scientifically inventive enough to warrant exclusivity, despite its clear medical advantages. But an Indian court overturned the decision last May — and that verdict in turn is now being contested.

Four of the lawsuits filed in February target other Indian patents on sofosbuvir and two related drugs, Gilead's velpatasvir (sold in combination with sofosbuvir under the name Epclusa) and Daklinza (daclatasvir) from Bristol-Myers Squibb in New York City. The fifth challenges Gilead's application to 